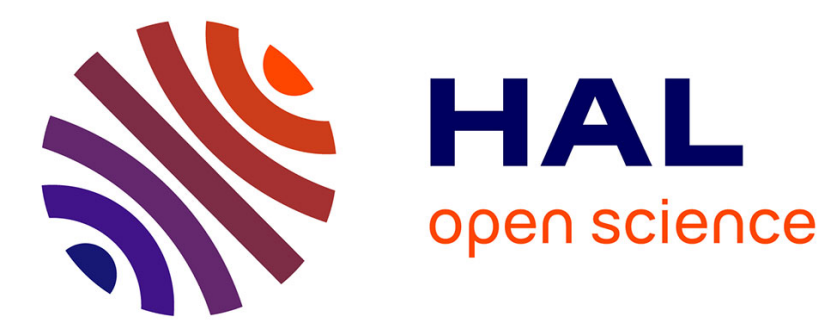

\title{
Ultrafast control of lattice strain via magnetic circular dichroism
}

Marwan Deb, Elena Popova, Steffen Peer Zeuschner, Wolfram Leitenberger, Niels Keller, Matthias Rössle, Matias Bargheer

\section{- To cite this version:}

Marwan Deb, Elena Popova, Steffen Peer Zeuschner, Wolfram Leitenberger, Niels Keller, et al.. Ultrafast control of lattice strain via magnetic circular dichroism. Physical Review B, 2021, 103, 10.1103/physrevb.103.064301 . hal-03439604

\section{HAL Id: hal-03439604 https://hal.science/hal-03439604}

Submitted on 22 Nov 2021

HAL is a multi-disciplinary open access archive for the deposit and dissemination of scientific research documents, whether they are published or not. The documents may come from teaching and research institutions in France or abroad, or from public or private research centers.
L'archive ouverte pluridisciplinaire HAL, est destinée au dépôt et à la diffusion de documents scientifiques de niveau recherche, publiés ou non, émanant des établissements d'enseignement et de recherche français ou étrangers, des laboratoires publics ou privés. 


\title{
Ultrafast control of lattice strain via magnetic circular dichroism
}

\author{
Marwan Deb $\odot,{ }^{1, *}$ Elena Popova $\odot,{ }^{2}$ Steffen Peer Zeuschner, ${ }^{1,3}$ Wolfram Leitenberger, ${ }^{1}$ Niels Keller, ${ }^{2}$ \\ Matthias Rössle $\odot,{ }^{3}$ and Matias Bargheer ${ }^{1,3}$ \\ ${ }^{1}$ Institut für Physik und Astronomie, Universität Potsdam, Karl-Liebknecht-Strasse 24-25, 14476 Potsdam, Germany \\ ${ }^{2}$ Groupe d'Etude de la Matière Condensée (GEMaC), CNRS UMR 8635, Université Paris-Saclay, 78035 Versailles, France \\ ${ }^{3}$ Helmholtz Zentrum Berlin für Materialien und Energie GmbH, Albert-Einstein-Strasse 15, 12489 Berlin, Germany
}

(Received 8 September 2020; accepted 22 January 2021; published 1 February 2021)

\begin{abstract}
Using ultrafast x-ray diffraction, we directly monitor the lattice dynamics induced by femtosecond laser pulses in nanoscale thin films of bismuth iron garnet in external magnetic fields $H_{\text {ext }}$. We control the ultrafast laserinduced lattice strain amplitude by changing the laser pulse helicity. The strength of $H_{\text {ext }}$ is used as an external parameter to switch the helicity dependence on and off, respectively. Based on magneto-optical spectroscopic measurements, we explain these phenomena by magnetic circular dichroism. Our findings highlight an important approach for ultrafast manipulation of lattice strain in magnetic materials, in particular insulators, and open exciting perspectives towards ultrafast control of lattice strain and heat-induced magnetization switching and spin waves in bismuth substituted iron garnets using the polarization of light.
\end{abstract}

DOI: 10.1103/PhysRevB.103.064301

Controlling the properties of matter has always been a major issue in condensed-matter physics. From a fundamental point of view, it requires a deep understanding of the interactions among spins, charge, and lattice degrees of freedom and their response to external stimuli. Traditionally, such control has been realized by changing external conditions such as temperature, pressure, and electric or magnetic fields [1,2]. Recently, intense ultrashort laser pulses have been proven to be an efficient tool for manipulating magnetic [3-5], electric [6,7], optic [8], and crystallographic [9-11] properties of materials on ultrashort time scales. The coupled dynamics is measured in real time with high spatiotemporal resolution using time-resolved pump-probe techniques, which are selective for one of the subsystems [3-11]. This unique opportunity provided by ultrafast laser pulses has led to the discovery of important phenomena for both fundamental science and technological applications, such as laser-induced room temperature superconductivity [12], inverted strain pulses [13,14], control of the ferroelectric [15] and magnetic $[3,4,16,17]$ order, and triggering coherent spin [4,18-20] and phonon [21,22] dynamics. Some of these phenomena are explained via processes involving the coupling and energy transfer between the degrees of freedom in solids $[3-5,12,14,20,23]$ and others are triggered by nonlinear optical processes like impulsive simulated Raman scattering $[4,5,16,18,19,21]$. These nonlinear optical processes can offer the advantage of controlling physical properties using the light polarization $[4,5,16,18,19]$. For linear absorption such control has been reported in very few cases [24-28]. In particular, the effect of magnetic circular dichroism has mainly been discussed in the context of helicity-dependent all-optical switching in metallic magnets [25,28].

*Corresponding author: madeb@uni-potsdam.de
In magnetic materials, one of the most important approaches used for ultrafast control of their properties is based on the interaction between the lattice and the magnetization. So far, this approach has attracted attention in two different ways: The first is related to controlling the magnetization dynamics with picosecond strain pulses generated in metallic film by femtosecond laser pulses [29-33]. It was demonstrated that a strain pulse can trigger a coherent magnetization precession in metal [29-31], semiconductor [32], and dielectric $[33,34]$ magnetic films. Because of its long propagation distance of several millimeters combined with low energy losses [35,36], strain pulses offer an important opportunity to excite magnetization precession in magnetic materials deeply embedded in opaque heterostructure devices [32-34]. The second way is related to control the lattice strain by the ultrafast modification of the magnetic order [14,23,37-41]. Most studies in this direction focus on measuring the lattice response upon the ultrafast demagnetization of a metallic magnet with femtosecond laser pulses [14,23,37-41]. The results of these investigations are explained by considering the transfer of angular momentum [37,40] and energy $[14,23,38,39]$ from the spins to the lattice. By now, these efforts have yielded great progress in the fundamental understanding of lattice-spin interactions on ultrashort time scales. However, an important question remains: Is it possible to use the magnetization for ultrafast manipulation of the lattice strain via an external parameter of the laser pulse like the state of its polarization? If yes, what is the fundamental mechanism behind this phenomenon? In addition, what can be the impact of such control on the ultrafast magnetization dynamics, especially in magnetic insulators?

In this paper, we use ultrafast $\mathrm{x}$-ray diffraction (UXRD) to directly monitor the lattice dynamics induced by a femtosecond laser excitation in nanoscale thin films of bismuth iron garnet under external magnetic fields, $H_{\text {ext }}$, applied 


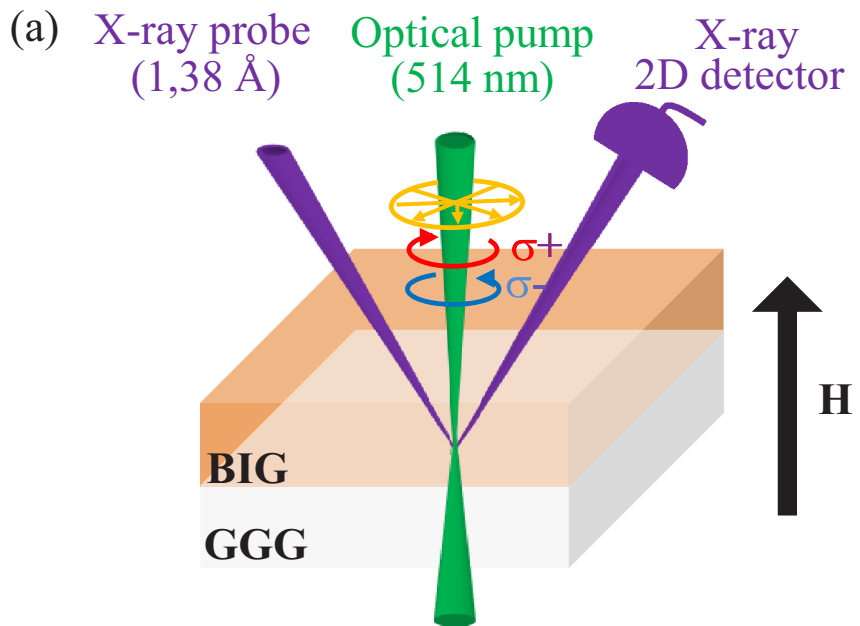

(b)

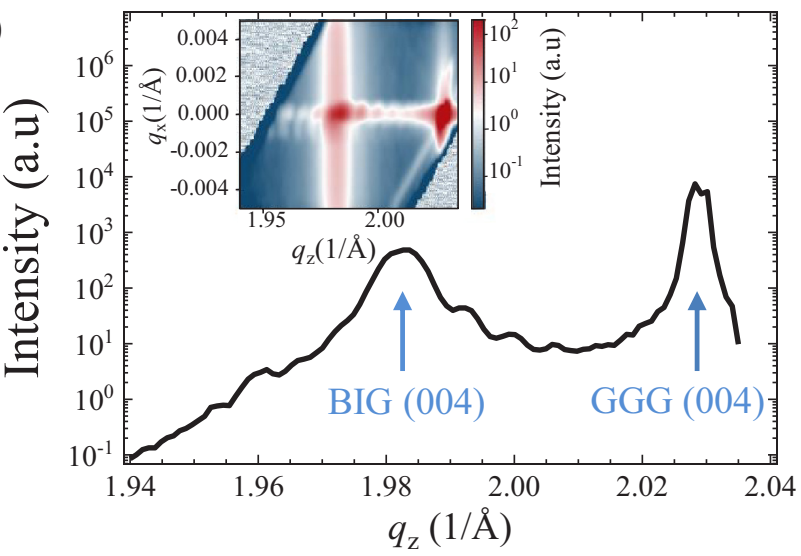

FIG. 1. Experimental configuration and static x-ray diffraction results. (a) Sketch of the UXRD configuration with pump pulses and external magnetic field perpendicular to the film. (b) X-ray diffraction line profile recorded along the $q_{z}$ direction around the 004 Bragg reflections of the BIG layer and the GGG substrate. The arrows indicate the 004 Bragg reflection related to BIG and the GGG. The inset shows the reciprocal space map around the 004 Bragg reflections of BIG layer and GGG substrate.

perpendicular to the film plane [see Fig. 1(a)]. By changing the helicity of the laser pulse, we demonstrate ultrafast manipulation of the lattice strain: A huge 20\% difference of the strain amplitude is controlled by the light helicities when the magnetization is saturated perpendicular to the film plane. In addition, we find that the lattice strain can be controlled by varying the strength of $H_{\mathrm{ext}}$. Both effects rely on ultrafast but long lived stress and the concomitant strain dynamics are only limited by the sound velocity. Based on magneto-optical spectroscopic measurements, we demonstrate that these phenomena can be explained considering the circular magnetic dichroism.

Our study is based on a 90 -nm thick bismuth iron garnet $\left(\mathrm{Bi}_{3} \mathrm{Fe}_{5} \mathrm{O}_{12}, \mathrm{BIG}\right)$ film grown by pulsed laser disposition onto a (100) gadolinium gallium garnet $\left(\mathrm{Gd}_{3} \mathrm{Ga}_{5} \mathrm{O}_{12}\right.$, GGG) substrate. A detailed description of the growth conditions can be found in Ref. [42]. The BIG material is a ferrimagnetic insulator with a high Curie temperature $\left(T_{\mathrm{C}} \sim\right.$ $680 \mathrm{~K})$ [43], large optical band gap $\left(E_{\mathrm{g}} \sim 2.4 \mathrm{eV}\right)[44,45]$ and giant magneto-optical Faraday rotation $(\sim 37 \mathrm{deg} / \mu \mathrm{m}$ at $2.3 \mathrm{eV}$ ) [46,47]. These combined properties make this material a promising candidate for nonreciprocal magneto-optical devices $[48,49]$. The UXRD measurements are performed at the XPP-KMC3 experimental station of the synchrotron radiation source BESSY II, Helmholtz-Zentrum Berlin (HZB), during the standard multibunch operation mode. In this operation mode, the bending magnet emits hard x-ray pulses with a duration of $\sim 100 \mathrm{ps}$. The sample is excited at normal incidence with a pump beam at the central wavelength of $514 \mathrm{~nm}$, obtained by frequency doubling the output of a $\mathrm{Yb}: \mathrm{KGW}$ laser system operating at $104 \mathrm{kHz}$ repetition rate and with $600-\mathrm{fs}$ pulse duration. At this pump wavelength, the BIG film absorbs about $38 \%$ of the incident energy. The pump pulse is focused onto the sample in a circular spot with a diameter of $560 \mu \mathrm{m}$, while the $\mathrm{x}$-ray probe has a rectangular shape with dimensions of $460 \times 150 \mu \mathrm{m}^{2}$. The $\mathrm{x}$-ray photon energy $9 \mathrm{keV}(\lambda=1.38 \AA$ ) was selected by a Si double crystal monochromator. The polarization of the pump beam is controlled using a quarter-wave plate (QWP). The external magnetic field $H_{\text {ext }}$ is applied perpendicular to the plane of the film. All measurements are performed at room temperature.

Before the UXRD measurements, we investigated the static crystalline properties of the BIG/GGG sample using the same $\mathrm{x}$-ray setup. Figure 1(b) shows the $\mathrm{x}$-ray diffraction line profile recorded along the $q_{z}$ direction under symmetric Bragg diffraction at $q_{x}=0$, where $q_{z}$ and $q_{x}$ represent the scattering wave vector components along the out-of-plane and the in-plane directions of the sample. The corresponding reciprocal space map of diffracted intensity as a function of $q_{z}$ and $q_{x}$ is shown in the inset of Fig. 1(b). We observe two well-separated diffraction peaks at $q_{z}=1.92 \AA^{-1}$ and $q_{z}=$ $2.03 \AA^{-1}$, corresponding to the 004 Bragg diffraction of BIG and GGG, respectively. The absence of any other diffraction peaks indicates the single-crystalline and single-phase nature the BIG film. This is in agreement with transmission electron microscopy measurements in BIG samples obtained in identical growth conditions [50]. The width of the BIG Bragg reflection $\Delta q_{z}=0.007 \AA^{-1}$ and the Laue oscillations indicate that the coherence length coincides with the film thickness. In the UXRD experiments, we measured the shift of this peak induced by laser excitation and we extracted via Bragg's law the corresponding lattice strain $\eta(t)$ as a function of the time delay $t$ between the pump and the probe pulses.

Figure 2 shows the lattice strain induced by right $\left(\sigma^{+}\right)$and left $\left(\sigma^{-}\right)$circularly polarized laser pulses with a fluence of $F_{\text {pump }}=3.5 \mathrm{~mJ} \mathrm{~cm}^{-2}$ at an external magnetic field of $H_{\mathrm{ext}}=$ $450 \mathrm{mT}$. At this pump fluence, the static optical heating of the sample is about $50 \mathrm{~K}$. This increases the global static temperature of the sample to $350 \mathrm{~K}$, which is very small compared to $T_{\mathrm{C}}(\mathrm{BIG}) \sim 680 \mathrm{~K}$. The lattice dynamics are governed by two distinct time scales. Within the first $150 \mathrm{ps}$ an ultrafast lattice expansion by $0.02 \%$ is observed. The signal is limited by the x-ray pulse duration. The time of maximum expansion is given by $T=d / v=17 \mathrm{ps}$, where $d$ is the thickness of the film and $v$ is the sound velocity of BIG [51]. The long-lived expanded state exhibits very slow relaxation dynamics. In the measured time window of $5 \mathrm{~ns}$, the strain amplitude decays only a little by heat transport to the substrate. The central observation depicted in Fig. 2 is the change of $\sim 20 \%$ in the 


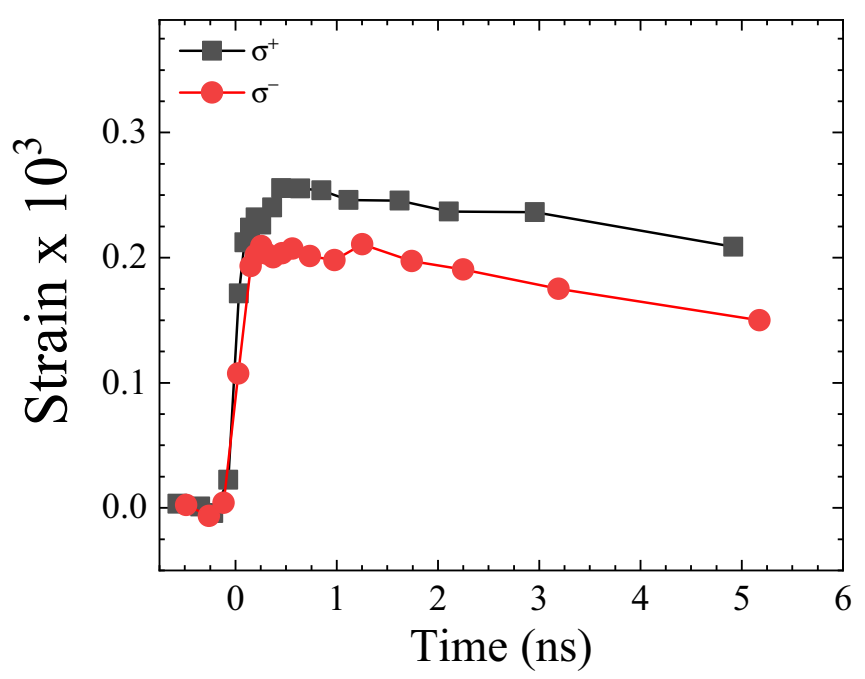

FIG. 2. Time-resolved lattice dynamics induced by right $\left(\sigma^{+}\right.$, squares) and left ( $\sigma^{-}$, solid circles) circular polarization pump beam with a pump fluence $F_{\text {pump }}=3.5 \mathrm{~mJ} \mathrm{~cm}^{-2}$ for $H_{\mathrm{ext}}=450 \mathrm{mT}$.

generated strain amplitude by reversing the helicity of the pump beam.

In order to study this phenomenon in more detail, we have measured the relative strain amplitude at different time delays of $t=500 \mathrm{ps}, t=1000 \mathrm{ps}$, and $t=2000 \mathrm{ps}$ as a function of the angle $\theta$ between the fast axis of the QWP and the p-polarized beam [see Figs. 3(a), 3(b), and 3(c)]. For all time delays, the strain amplitude follows a clear sinu-

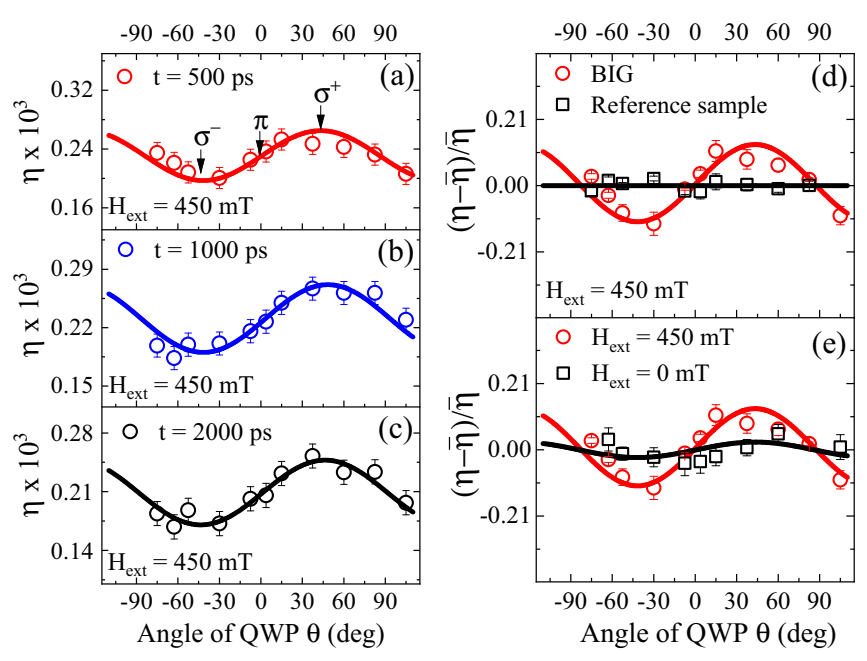

FIG. 3. Strain amplitude $\eta$ measured as a function of orientation angle $\theta$ of the quarter wave plate for $F_{\text {pump }}=3.5 \mathrm{~mJ} \mathrm{~cm}^{-2}$. The absolute strain $\eta(\theta)$ obtained for BIG at three different time delays of (a) $t=500 \mathrm{ps}$, (b) $t=1000 \mathrm{ps}$, and (c) $t=2000 \mathrm{ps}$ for $H_{\mathrm{ext}}=$ $450 \mathrm{mT}$. (d) The relative strain $(\eta(\theta)-\bar{\eta}) / \bar{\eta}$ obtained for BIG and the nonmagnetic reference sample at $t=500 \mathrm{ps}$ for $H_{\mathrm{ext}}=450 \mathrm{mT}$. (e) The relative strain for BIG at $t=500 \mathrm{ps}$ for $H_{\mathrm{ext}}=450 \mathrm{mT}$ and $H_{\text {ext }}=0 \mathrm{mT}$. The arrows in (a) highlight the QWP angles corresponding to circular right $(\sigma+)$, linear $(\pi)$, and circular left $(\sigma+)$ polarizations of the pump beam. The solid lines are guides to the eye. The error bars indicate the standard deviation of the time resolved data. soidal form with a periodicity of $180^{\circ}$. The minimum strain is observed for the left circular polarization $\sigma^{+}(\theta=+45)$, while its minimum is observed for the right circular polarization $\sigma^{-}(\theta=-45)$. The $p$ - or $s$ - polarized pump beam $\pi(\theta=0$ and \pm 90$)$ generate the same strain amplitude that is approximately the average of those induced by $\sigma^{+}$and $\sigma^{-}$ polarizations, i.e., $\eta(\pi)=\left(\eta\left(\sigma^{+}\right)+\eta\left(\sigma^{-}\right)\right) / 2$. In Fig. 3(d) we cross check this finding by comparing the helicity dependence of the photoinduced strain amplitude in BIG to the helicity dependence observed under the very same excitation conditions in $\mathrm{SrRuO}_{3}$ thin film that is nonmagnetic at room temperature and is used as a standard reference sample for finding the zero time delay in pump-probe experiments at the XPP-KMC3 beamline [52,53]. Clearly, the strain amplitude for the nonmagnetic reference sample [black in Fig. 3(d)] is independent of the pump polarization at $450 \mathrm{mT}$, where the strain in BIG shows a strong modulation. More importantly, we find that the helicity-dependent strain modulation generated in BIG can be nearly switched off by setting $H_{\text {ext }}=0$. The black symbols in Fig. 3(e) show nearly the same strain for any rotation angle of the QWP.

To give a comprehensive understanding of these phenomena, it is important to clarify first the mechanism of strain generation in BIG thin films by near-band-gap laser excitation. Qualitatively, such processes can be described in the following way. The light energy is absorbed in the BIG layer by electronic transitions related to the $\mathrm{Fe}^{3+}$ ions $[46,54,55]$. These electronic states are very short lived [51] and rapidly transfer the entire energy to vibrations of the lattice via electron-phonon coupling. This lattice temperature rise associated with this heat energy induces a lattice expansion, which is directly quantified by UXRD measurements. We note that the demagnetization dynamics in iron garnets is a very slow process that occurs on the nanosecond time scale [56]. Therefore, the magnetostrictive strain should have a very small or no contribution to the lattice dynamics measured in the first $50 \mathrm{ps}$, where the coherent and incoherent phonons are already generated. The heat in the BIG layer is mainly transported to the GGG substrate via phonon heat conduction. This process is characterized by an extremely long time scale (see Fig. 2), which is in good agreement with the low thermal conductivity in iron garnets [51,57].

From the above-described processes that lead to the lattice strain observed in Figs. 2 and 3, we can conclude that the origin of the polarization-dependent lattice dynamics should satisfy the following three fundamental criteria: (i) the absorption of light depends on the state of its polarization, (ii) occurs only in magnetic materials, and (iii) depends on the strength of $H_{\text {ext }}$, i.e., the amplitude of the magnetization. These criteria are fulfilled by the magnetic circular dichroism (MCD), which is manifested for a given direction of the magnetization by a variation in the light absorption when going over from right to left circular polarization [58].

In order to confirm the physical origin behind the helicity-dependent lattice strain dynamics, we investigated the wavelength- and magnetic-field dependence of the MCD, which corresponds to the difference in absorption between the right and left circular polarization [45,59]. The measurements are carried out using a magneto-optical spectrometer based on $90^{\circ}$ polarization-modulation technique. A detailed description 

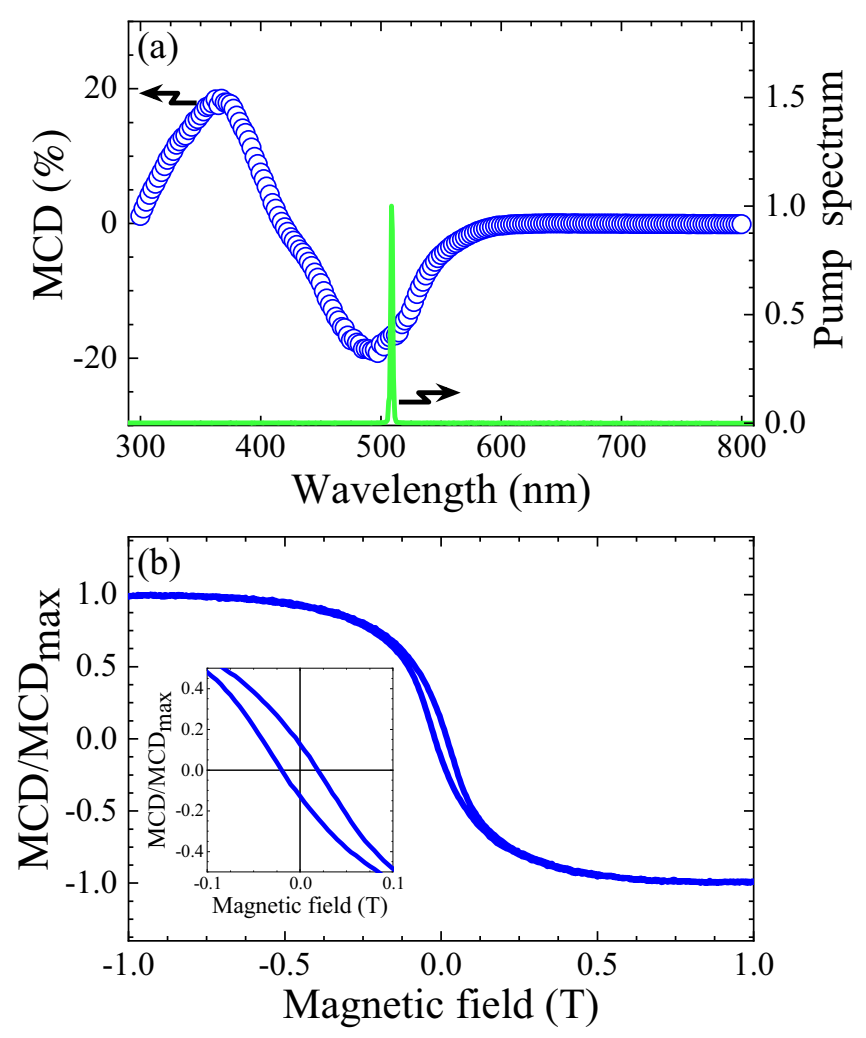

FIG. 4. Static magneto-optical and magnetic properties of the BIG film measured in polar configuration. (a) Magnetic circular dichroism spectrum of the BIG film, including the spectrum of the pump beam used in the UXRD measurements (green line). (b) Magnetic circular dichroism hysteresis loop. The inset shows a magnified view of the region round $0 \mathrm{~T}$.

of the experimental setup has been given in Ref. [60]. Briefly, the white light emitted by a $150-\mathrm{W}$ Xe arc lamp is polarized by a Rochon polarizer and modulated at a high frequency of $50 \mathrm{kHz}$ by a photoelectric modulator (PEM). The modulated light is focused onto the sample at normal incidence. The transmitted light is collimated and analyzed with a Rochon analyzer, and focused into a spectrometer. The MCD is deduced from the signal measured by a photomultiplier detector signal that is analyzed by a lock-in amplifier referenced to the first harmonic of the PEM. Figure 4 shows the spectral dependence of MCD measured over a wide range of wavelength between 300 and $800 \mathrm{~nm}$ with a saturating $H_{\text {ext }}$ applied perpendicular to the film plane. The MCD reaches a large value of $\sim-20 \%$ at $\lambda=514 \mathrm{~nm}$, which is the wavelength of the pump pulses used in UXRD experiments [see Fig. 4(a)]. This large difference of $\sim 20 \%$ in the absorption between the opposite light helicities confirms the proposed origin behind the polarization dependent lattice strain. It also reveals that the one photon absorption process is the dominant mechanism at $514 \mathrm{~nm}$, which is the expected result with a near-band-gap laser excitation. We note that the measured MCD is about 13 times higher than the one in GdFeCo [25], which is expected to be at the origin of the helicity-dependent all-optical switching in metallic magnets $[25,28]$. From a fundamental point of view, the large values of MCD in BIG can be explained by the large spin-orbit induced in Fe-3d orbitals due to their hybridization with the $\mathrm{Bi}-3 p$ ones, which are characterized by a huge spin-orbit coupling $[54,61]$. This mechanism is supported by cluster molecular-orbital theory [62,63] and band-structure calculations [64]. On the other hand, to reveal the origin of the small signal modulation observed at zero external magnetic field [Fig. 3(e)], we measured the polar MCD hysteresis loop [see Fig. 4(b)]. It shows a clear remanent magnetization $M_{\mathrm{r}} \sim 0.13 M_{\mathrm{s}}$ that is about $20 \%$ of the MCD signal for $H_{\mathrm{ext}}=$ $450 \mathrm{mT}$. This is in good agreement with the remaining strain modulation at $H_{\mathrm{ext}}=0 \mathrm{mT}$ reported in Fig. 3(e) compared to the modulation of the strain amplitude at $H_{\text {ext }}=450 \mathrm{mT}$. We note that this remnant magnetization corresponds to the average projection of the magnetic domains on the direction perpendicular to the film plane. These domains have a very small size $\left(\sim 0.2 \mu \mathrm{m}^{2}\right)[42,65]$, which guarantees that hundreds of thousands of them are located within the x-ray probe. On the other hand, for $H_{\mathrm{ext}}=450 \mathrm{mT}$, the magnetization of BIG is almost saturated perpendicular to the film plane and the difference in strain amplitude generated by the opposite helicity of light reaches a large value of $\sim 20 \%$.

These findings should not be restricted to BIG, but occur in all magnetic materials with good magneto-optical properties. From a practical point of view, light polarization (helicity) can thus be used to control any ultrafast phenomena that are influenced by the lattice strain (or lattice heating). For example, in Bi-substituted iron garnets, it was recently demonstrated that an ultrafast heating of lattice can excite high frequency spin waves $[20,66,67]$ and induce a full magnetization switching [68]. By choosing the appropriate pump wavelength to maximize the effect of MCD on lattice heating, it will be therefore possible to use the polarization of light in order to: (i) control the amplitude of the spin waves and (ii) obtain a helicity-dependent optical magnetization switching in magnetic insulators. Due to the low damping [49,69], large magneto-optical effect [46,47,49,70,71], and good transparency in the visible and infrared region [49,70,71] of bismuth substituted iron garnets, these phenomena are important for future data storage and processing technologies.

In summary, we demonstrated that femtosecond laser pulses can induce helicity-dependent ultrafast lattice dynamics in nanoscale thin films of BIG. We demonstrated a huge long-lived $20 \%$ difference in the lattice strain generated by the opposite light helicities when the magnetization is saturated perpendicular to the film plane. For both right and left circularly polarized light excitation, a variation of the external magnetic field strength can control the lattice strain amplitude. Based on magneto-optical spectroscopic measurements, we demonstrate that these phenomena can be explained by considering the MCD. Our findings open different perspectives on ultrafast manipulation of lattice strain and heat in magnetic materials, which allows for controlling magnetization switching and spin wave excitations in bismuth substituted iron garnets via the polarization of the laser pulse.

We acknowledge funding by the BMBF Project Grant No. 05K16IPA and M.D. acknowledges the Alexander von Humboldt Foundation for financial support. 
[1] C. Kittel, Introduction to Solid State Physics (Wiley, New York, 1986).

[2] W. Martienssen and H. Warlimont, Springer Handbook of Condensed Matter and Materials Data (Springer Science \& Business Media, Berlin,2006).

[3] E. Beaurepaire, J. C. Merle, A. Daunois, and J. Y. Bigot, Ultrafast Spin Dynamics in Ferromagnetic Nickel, Phys. Rev. Lett. 76, 4250 (1996).

[4] J.-Y. Bigot, W. Hübner, T. Rasing, and R. Chantrell, Ultrafast Magnetism I (Springer, Berlin, 2016).

[5] A. Kirilyuk, A. V. Kimel, and T. Rasing, Ultrafast optical manipulation of magnetic order, Rev. Mod. Phys. 82, 2731 (2010).

[6] R. Atanasov, A. Haché, J. L. P. Hughes, H. M. van Driel, and J. E. Sipe, Coherent Control of Photocurrent Generation in Bulk Semiconductors, Phys. Rev. Lett. 76, 1703 (1996).

[7] A. Schiffrin, T. Paasch-Colberg, N. Karpowicz, V. Apalkov, D. Gerster, S. Mühlbrandt, M. Korbman, J. Reichert, M. Schultze, S. Holzner et al. Optical-field-induced current in dielectrics, Nature (London) 493, 70 (2013).

[8] L. Le Guyader, A. Kirilyuk, T. Rasing, G. A. Wurtz, A. V. Zayats, P. F. A. Alkemade, and I. I. Smolyaninov, Coherent control of surface plasmon polariton mediated optical transmission, J. Phys. D 41, 195102 (2008).

[9] M. Bargheer, N. Zhavoronkov, Y. Gritsai, J. C. Woo, D. S. Kim, M. Woerner, and T. Elsaesser, Coherent atomic motions in a nanostructure studied by femtosecond $\mathrm{x}$-ray diffraction, Science 306, 1771 (2004)

[10] C. V. Korff Schmising, M. Bargheer, M. Kiel, N. Zhavoronkov, M. Woerner, T. Elsaesser, I. Vrejoiu, D. Hesse, and M. Alexe, Coupled Ultrafast Lattice and Polarization Dynamics in Ferroelectric Nanolayers, Phys. Rev. Lett. 98, 257601 (2007).

[11] M. Bargheer, N. Zhavoronkov, M. Woerner, and T. Elsaesser, Recent progress in ultrafast X-ray diffraction, Chem. Phys. Chem. 7, 783 (2006).

[12] D. Fausti, R. I. Tobey, N. Dean, S. Kaiser, A. Dienst, M. C. Hoffmann, S. Pyon, T. Takayama, H. Takagi, and A. Cavalleri, Light-induced superconductivity in a stripe-ordered cuprate, Science 331, 189 (2011).

[13] J. Pudell, A. von Reppert, D. Schick, F. Zamponi, M. Rössle, M. Herzog, H. Zabel, and M. Bargheer, Ultrafast negative thermal expansion driven by spin disorder, Phys. Rev. B 99, 094304 (2019).

[14] A. V. Reppert, M. Mattern, J.-E. Pudell, S. P. Zeuschner, K. Dumesnil, and M. Bargheer, Unconventional picosecond strain pulses resulting from the saturation of magnetic stress within a photoexcited rare earth layer, Struct. Dyn. 7, 024303 (2020).

[15] R. Mankowsky, A. von Hoegen, M. Först, and A. Cavalleri, Ultrafast Reversal of the Ferroelectric Polarization, Phys. Rev. Lett. 118, 197601 (2017).

[16] C. D. Stanciu, F. Hansteen, A. V. Kimel, A. Kirilyuk, A. Tsukamoto, A. Itoh, and T. Rasing, All-Optical Magnetic Recording with Circularly Polarized Light, Phys. Rev. Lett. 99, 047601 (2007).

[17] C.-H. Lambert, S. Mangin, B. S. D. C. S. Varaprasad, Y. K. Takahashi, M. Hehn, M. Cinchetti, G. Malinowski, K. Hono, Y. Fainman, M. Aeschlimann et al. All-optical control of ferromagnetic thin films and nanostructures, Science 345, 1337 (2014).
[18] F. Hansteen, A. Kimel, A. Kirilyuk, and T. Rasing, Femtosecond Photomagnetic Switching of Spins in Ferrimagnetic Garnet Films, Phys. Rev. Lett. 95, 047402 (2005).

[19] A. V. Kimel, A. Kirilyuk, P. A. Usachev, R. V. Pisarev, A. M. Balbashov, and T. Rasing, Ultrafast nonthermal control of magnetization by instantaneous photomagnetic pulses, Nature (London) 435, 655 (2005).

[20] M. Deb, E. Popova, M. Hehn, N. Keller, S. Petit-Watelot, M. Bargheer, S. Mangin, and G. Malinowski, Femtosecond Laser-Excitation-Driven High Frequency Standing Spin Waves in Nanoscale Dielectric Thin Films of Iron Garnets, Phys. Rev. Lett. 123, 027202 (2019).

[21] H. Katsuki, J. C. Delagnes, K. Hosaka, K. Ishioka, H. Chiba, E. S. Zijlstra, M. E. Garcia, H. Takahashi, K. Watanabe, M. Kitajima et al. All-optical control and visualization of ultrafast two-dimensional atomic motions in a single crystal of bismuth, Nat Commun. 4, 2801 (2013).

[22] M. Lejman, G. Vaudel, I. C. Infante, P. Gemeiner, V. E. Gusev, B. Dkhil, and P. Ruello, Giant ultrafast photo-induced shear strain in ferroelectric BiFeO3, Nat. Commun. 5, 4301 (2014).

[23] A. v. Reppert, J. Pudell, A. Koc, M. Reinhardt, W. Leitenberger, K. Dumesnil, F. Zamponi, and M. Bargheer, Persistent nonequilibrium dynamics of the thermal energies in the spin and phonon systems of an antiferromagnet, Struct. Dyn. 3, 054302 (2016).

[24] F. Hansteen, A. Kimel, A. Kirilyuk, and T. Rasing, Nonthermal ultrafast optical control of the magnetization in garnet films, Phys. Rev. B 73, 014421 (2006).

[25] A. R. Khorsand, M. Savoini, A. Kirilyuk, A. V. Kimel, A. Tsukamoto, A. Itoh, and T. Rasing, Role of Magnetic Circular Dichroism in All-Optical Magnetic Recording, Phys. Rev. Lett. 108, 127205 (2012).

[26] A. Stupakiewicz, K. Szerenos, D. Afanasiev, A. Kirilyuk, and A. V. Kimel, Ultrafast nonthermal photomagnetic recording in a transparent medium, Nature (London) 542, 71 (2017).

[27] A. Stupakiewicz, K. Szerenos, M. D. Davydova, K. A. Zvezdin, A. K. Zvezdin, A. Kirilyuk, and A. V. Kimel, Selection rules for all-optical magnetic recording in iron garnet, Nat. Commun. 10, 612 (2019).

[28] Y. Quessab, M. Deb, J. Gorchon, M. Hehn, G. Malinowski, and S. Mangin, Resolving the role of magnetic circular dichroism in multishot helicity-dependent all-optical switching, Phys. Rev. B 100, 024425 (2019).

[29] J.-W. Kim, M. Vomir, and J.-Y. Bigot, Ultrafast Magnetoacoustics in Nickel Films, Phys. Rev. Lett. 109, 166601 (2012).

[30] J.-W. Kim, M. Vomir, and J.-Y. Bigot, Controlling the spins angular momentum in ferromagnets with sequences of picosecond acoustic pulses, Sci. Rep. 5, 8511 (2015).

[31] T. L. Linnik, V. N. Kats, J. Jäger, A. S. Salasyuk, D. R. Yakovlev, A. W. Rushforth, A. V. Akimov, A. M. Kalashnikova, M. Bayer, and A. V. Scherbakov, The effect of dynamical compressive and shear strain on magnetic anisotropy in a low symmetry ferromagnetic film, Phys. Scripta 92, 054006 (2017).

[32] A. V. Scherbakov, A. S. Salasyuk, A. V. Akimov, X. Liu, M. Bombeck, C. Brüggemann, D. R. Yakovlev, V. F. Sapega, J. K. Furdyna, and M. Bayer, Coherent Magnetization Precession in Ferromagnetic (Ga,Mn)As Induced by Picosecond Acoustic Pulses, Phys. Rev. Lett. 105, 117204 (2010).

[33] M. Deb, E. Popova, M. Hehn, N. Keller, S. Mangin, and G. Malinowski, Picosecond acoustic-excitation-driven ultrafast 
magnetization dynamics in dielectric Bi-substituted yttrium iron garnet, Phys. Rev. B 98, 174407 (2018).

[34] M. Deb, E. Popova, S. P. Zeuschner, M. Hehn, N. Keller, S. Mangin, G. Malinowski, and M. Bargheer, Generation of spin waves via spin-phonon interaction in a buried dielectric thin film, Phys. Rev. B 103, 024411 (2021).

[35] H. Y. Hao and H. J. Maris, Study of Phonon Dispersion in Silicon and Germanium at Long Wavelengths Using Picosecond Ultrasonics, Phys. Rev. Lett. 84, 5556 (2000).

[36] O. L. Muskens and J. I. Dijkhuis, High Amplitude, Ultrashort, Longitudinal Strain Solitons in Sapphire, Phys. Rev. Lett. 89, 285504 (2002).

[37] E. Jal, V. López-Flores, N. Pontius, T. Ferté, N. Bergeard, C. Boeglin, B. Vodungbo, J. Lüning, and N. Jaouen, Structural dynamics during laser-induced ultrafast demagnetization, Phys. Rev. B 95, 184422 (2017).

[38] A. Koc, M. Reinhardt, A. von Reppert, M. Rössle, W. Leitenberger, M. Gleich, M. Weinelt, F. Zamponi, and M. Bargheer, Grueneisen-approach for the experimental determination of transient spin and phonon energies from ultrafast x-ray diffraction data: Gadolinium, J. Phys.: Condens. Matter 29, 264001 (2017).

[39] A. H. Reid, X. Shen, P. Maldonado, T. Chase, E. Jal, P. W. Granitzka, K. Carva, R. K. Li, J. Li, L. Wu et al. Beyond a phenomenological description of magnetostriction, Nat. Commun. 9, 388 (2018).

[40] C. Dornes, Y. Acremann, M. Savoini, M. Kubli, M. J. Neugebauer, E. Abreu, L. Huber, G. Lantz, C. A. F. Vaz, H. Lemke et al. The ultrafast Einstein-de Haas effect, Nature (London) 565, 209 (2019).

[41] A. von Reppert, L. Willig, J.-E. Pudell, S. P. Zeuschner, G. Sellge, F. Ganss, O. Hellwig, J. A. Arregi, V. Uhlî́, A. Crut et al. Spin stress contribution to the lattice dynamics of FePt, Sci. Adv. 6, eaba1142 (2020).

[42] E. Popova, L. Magdenko, H. Niedoba, M. Deb, B. Dagens, B. Berini, M. Vanwolleghem, C. Vilar, F. Gendron, A. Fouchet et al. Magnetic properties of the magnetophotonic crystal based on bismuth iron garnet, J. Appl. Phys. 112, 093910 (2012).

[43] B. Vertruyen, R. Cloots, J. S. Abell, T. J. Jackson, R. C. da Silva, E. Popova, and N. Keller, Curie temperature, exchange integrals, and magneto-optical properties in off-stoichiometric bismuth iron garnet epitaxial films, Phys. Rev. B 78, 094429 (2008).

[44] S. Kahl, V. Popov, and A. M. Grishin, Optical transmission and faraday rotation spectra of a bismuth iron garnet film, J. Appl. Phys. 94, 5688 (2003)

[45] M. Deb, E. Popova, A. Fouchet, and N. Keller, Full spin polarization of complex ferrimagnetic bismuth iron garnet probed by magneto-optical Faraday spectroscopy, Phys. Rev. B 87, 224408 (2013).

[46] M. Deb, E. Popova, A. Fouchet, and N. Keller, Magnetooptical faraday spectroscopy of completely bismuth-substituted $\mathrm{Bi}_{3} \mathrm{Fe}_{5} \mathrm{O}_{12}$ garnet thin films, J. Phys. D 45, 455001 (2012).

[47] M. Deb, E. Popova, and N. Keller, Different magneto-optical response of magnetic sublattices as a function of temperature in ferrimagnetic bismuth iron garnet films, Phys. Rev. B 100, 224410 (2019).

[48] E. Popova, A. F. F. Galeano, M. Deb, B. Warot-Fonrose, H. Kachkachi, F. Gendron, F. Ott, B. Berini, and N. Keller,
Magnetic anisotropies in ultrathin bismuth iron garnet films, J. Magn. Magn. Mater. 335, 139 (2013).

[49] G. D. Winkler, Magnetic Garnets (Vieweg, Braunschweig, Germany, 1981).

[50] E. Popova, M. Deb, L. Bocher, A. Gloter, O. Stéphan, B. Warot-Fonrose, B. Berini, Y. Dumont, and N. Keller, Interplay between epitaxial strain and low dimensionality effects in a ferrimagnetic oxide, J. Appl. Phys. 121, 115304 (2017).

[51] S. P. Zeuschner, J. E. Pudell, A. von Reppert, M. Deb, E. Popova, N. Keller, M. Rössle, M. Herzog, and M. Bargheer, Measurement of transient strain induced by two-photon excitation, Phys. Rev. Res. 2, 022013(R) (2020).

[52] H. A. Navirian, D. Schick, P. Gaal, W. Leitenberger, R. Shayduk, and M. Bargheer, Thermoelastic study of nanolayered structures using time-resolved $\mathrm{x}$-ray diffraction at high repetition rate, Appl. Phys. Lett. 104, 021906 (2014).

[53] M. Reinhardt, A. Koc, W. Leitenberger, P. Gaal, and M. Bargheer, Optimized spatial overlap in optical pump-X-ray probe experiments with high repetition rate using laser-induced surface distortions, J. Synchrotron Radiat. 23, 474 (2016).

[54] G. F. Dionne and G. A. Allen, Molecular-orbital analysis of magneto-optical Bi-O-Fe hybrid excited states, J. Appl. Phys. 75, 6372 (1994).

[55] F. Iori, A. Teurtrie, L. Bocher, E. Popova, N. Keller, O. Stéphan, and A. Gloter, Bismuth iron garnet: Ab initio study of electronic properties, Phys. Rev. B 100, 245150 (2019).

[56] L. A. Shelukhin, V. V. Pavlov, P. A. Usachev, P. Y. Shamray, R. V. Pisarev, and A. M. Kalashnikova, Ultrafast laser-induced changes of the magnetic anisotropy in a low-symmetry iron garnet film, Phys. Rev. B 97, 014422 (2018).

[57] N. P. Padture and P. G. Klemens, Low thermal conductivity in garnets, J. Am. Ceram. Soc. 80, 1018 (1997).

[58] D. Gignoux, M. Schlenker, and É. D. T. de Lacheisserie, Magnetism: Fundamentals (Springer, New York, 2005).

[59] M. Kucera, J. Bok, and K. Nitsch, Faraday rotation and MCD in Ce doped YIG, Solid State Commun. 69, 1117 (1989).

[60] M. Deb, M. Vomir, J.-L. Rehspringer, and J.-Y. Bigot, Ultrafast optical control of magnetization dynamics in polycrystalline bismuth doped iron garnet thin films, Appl. Phys. Lett. 107, 252404 (2015).

[61] S. Wittekoek and D. E. Lacklison, Investigation of the Origin of the Anomalous Faraday Rotation of $\mathrm{Bi}_{x} \mathrm{Ca}_{3-x} \mathrm{Fe}_{3.5+0.5 x} \mathrm{~V}_{1.5-0.5 x} \mathrm{O}_{12}$ by Means of the Magnetooptical Kerr Effect, Phys. Rev. Lett. 28, 740 (1972).

[62] A. V. Zenkov and A. S. Moskvin, Bismuth-induced increase of the magneto-optical effects in iron garnets: A theoretical analysis, J. Phys.: Condens. Matter 14, 6957 (2002).

[63] S. Sugano and N. Kojima, Magneto-optics (Springer Science \& Business Media, Berlin, 2013), Vol. 128.

[64] T. Oikawa, S. Suzuki, and K. Nakao, First-principles study of spin-orbit interactions in bismuth iron garnet, J. Phys. Soc. Jpn. 74, 401 (2005).

[65] E. Popova, A. Shengelaya, D. Daraselia, D. Japaridze, S. Cherifi-Hertel, L. Bocher, A. Gloter, O. Stéphan, Y. Dumont, and N. Keller, Bismuth iron garnet Bi3Fe5O12: A room temperature magnetoelectric material, Appl. Phys. Lett. 110, 142404 (2017).

[66] M. Deb, E. Popova, M. Hehn, N. Keller, S. Petit-Watelot, M. Bargheer, S. Mangin, and G. Malinowski, Damping of Standing Spin Waves in Bismuth-Substituted Yttrium Iron Garnet 
as Seen via the Time-Resolved Magneto-Optical Kerr Effect, Phys. Rev. Appl. 12, 044006 (2019).

[67] Y. Hashimoto, D. Bossini, T. H. Johansen, E. Saitoh, A. Kirilyuk, and T. Rasing, Frequency and wavenumber selective excitation of spin waves through coherent energy transfer from elastic waves, Phys. Rev. B 97, 140404(R) (2018).

[68] C. S. Davies, K. H. Prabhakara, M. D. Davydova, K. A. Zvezdin, T. B. Shapaeva, S. Wang, A. K. Zvezdin, A. Kirilyuk, T. Rasing, and A. V. Kimel, Anomalously Damped Heat-
Assisted Route for Precessional Magnetization Reversal in an Iron Garnet, Phys. Rev. Lett. 122, 027202 (2019).

[69] L. Soumah, N. Beaulieu, L. Qassym, C. Carrétéro, E. Jacquet, R. Lebourgeois, J. Ben Youssef, P. Bortolotti, V. Cros, and A. Anane, Ultra-low damping insulating magnetic thin films get perpendicular, Nat. Commun. 9, 3355 (2018).

[70] P. Hansen and J. P. Krumme, Magnetic and magneto-optical properties of garnet films, Thin Solid Films 114, 69 (1984).

[71] G. F. Dionne, Magnetic Oxides (Springer, New York, 2009). 